\title{
Postoperative Physical Therapy Following Coronary Artery Bypass Surgery - A Case Report
}

\author{
Tushar Ubare $^{1}$, Namrata Rathi ${ }^{2}$, Leena Jaiswal ${ }^{3}$ \\ ${ }^{1}$ Professor, Shri K. R. Pandav College of Physiotherapy, Bhilewada, Bhandara, India. \\ ${ }^{2}$ Professor, Department of Neurosciences Physiotherapy, Shri K. R. Pandav College of Physiotherapy, \\ Bhilewada, Bhandara, India. \\ ${ }^{3}$ Assistant Professor, Smt. Radhikatai Pandav College of Physiotherapy, Nandanvan, Nagpur. \\ Corresponding Author: Namrata Rathi
}

DOI: https://doi.org/10.52403/ijhsr.20220319

\begin{abstract}
One of the most prevalent consequences following coronary artery bypass graft (CABG) surgery is pulmonary difficulties. However, not all patients are at the same risk of pulmonary complications after surgery (PPCs) (1). Postoperative pulmonary issues are the most common problems discovered and treated following abdominal or cardiothoracic surgery (PPCs) (1). Patients with a history of lung disease had a higher risk of problems after surgery, according to research. Treatment for coronary artery disease (CAD) aims to reduce or eliminate the disease's repercussions, as well as its morbidity and death (2). Treatment for coronary artery disease (CAD) tries to lessen or eliminate the illness's consequences, including morbidity and mortality(3). Atelectasis is a common complication of coronary artery bypass surgery. Atelectasis can be caused by general anaesthesia, diaphragmatic dysfunction, abdominal distension, chest wall changes, pleural effusions, and discomfort (4). Physiotherapists have typically employed different respiratory therapies after coronary artery bypass graft surgery $(\mathrm{CABG})$ to reduce the occurrence of postoperative pulmonary issues (PPC) (5). Despite its widespread use, the effectiveness of any particular chest physical therapy is unknown. Every day, patients with coronary artery disease around the world undergo coronary artery bypass graft (CABG) surgery(6). Despite advances in anesthetic, cardiopulmonary bypass procedures, and pre-and postoperative care, CABG continues to be associated with a high prevalence of pulmonary complications (PPC). In the postoperative phase after CABG, respiratory physiotherapy is advised to improve lung function and prevent or treat pulmonary problems (7).
\end{abstract}

Keywords: Coronary artery bypass graft surgery, Postoperative pulmonary complications, Preoperative risk factors, Physiotherapy rehabilitation.

\section{INTRODUCTION}

Pulmonary difficulties are one of the most commonly reported complications following coronary artery bypass graft (CABG) surgery (1). Spirometry with incentives is frequently utilized as a part of postoperative pulmonary care (2). The goal of coronary artery disease (CAD) treatment is to lower morbidity and mortality by reducing or preventing the illness's consequences (3). The most common cause of morbidity and mortality after cardiac
CABG surgery is pulmonary dysfunction and its consequences (3). A sternotomy, pleurotomy caused by the insertion of the left internal thoracic artery, pleural drain insertion, diaphragmatic dysfunction caused by viscera manipulation, and phrenic nerve reflex dysfunction caused by the administration of cold cardioplegic solution are all examples of pulmonary function impairment (3). Physiotherapy, such as incentive spirometry and respiratory muscle training, has been proven in numerous trials 
to be useful in lowering pulmonary dysfunction throughout the preoperative and postoperative periods (3). Both with and without extracorporeal circulation, coronary bypass surgery compromises the respiratory system mechanically (4). Off-pump When compared to $\mathrm{CABG}$ surgery with $\mathrm{CPB}$, CABG surgery does not provide better protection against postoperative pulmonary impairment. Preoperative physical therapy has been shown to reduce postoperative radiological anomalies, auscultation, blood gases, and improve quality of life in patients undergoing both abdominal and open-heart surgery (4). As a result, prescription walking and/or thoracic mobility exercises during inpatient or Phase 1 cardiac rehabilitation (CR) may be more suitable to restoring pre-operative physical functional capacity (5). Early mobilization, physical therapy interventions, and a variety of respiratory procedures such as deep breathing, coughing, or incentive spirometry have traditionally been used to prevent and cure atelectasis (6). These therapies have been aimed at mobilization of secretions or increasing functional residual capacity. Recent research has demonstrated that early postoperative mobilization is just as helpful as chest physical therapy and respiratory exercises in preventing postoperative atelectasis or pulmonary problems (6). In the postoperative period following $\mathrm{CABG}$, respiratory physiotherapy has been advocated to improve lung function and prevent or treat pulmonary problems (7). CABG is associated with a high rate of postoperative problems, which may be linked to a longer hospital stay (8). Smoking is linked to both coronary artery disease and pulmonary disease, and many CABG patients have pulmonary disease as evidenced by spirometry (2). When compared to saphenous vein conduit bypass, the use of the internal mammary artery (IMA) for coronary artery bypass graft (CABG) surgery enhances the percentage of PC (4). A therapeutic benefit of this early 'walking fitness' is the potential for earlier gains in functional activity and faster referral to an exercise-based outpatient (Phase 2) cardiac rehabilitation (CR) programme' (5). Following first-time, elective CABG, a physiotherapy-supervised, organized walking program in the inpatient environment significantly increases submaximal exercise capacity at release (5). Chest X-rays were interpreted as having minimal atelectasis (no atelectasis or one segment involvement) or as having marked atelectasis (lobar atelectasis or two separate areas of segmental atelectasis) (6). Patients anticipating CABG had a shorter stay in the hospital, a higher quality of life, and lower anxiety scores after pre-and postoperative rehabilitation, according to studies (8). After CABG, respiratory physical therapy is commonly administered in most institutions in the hopes of reducing postoperative problems (8). Hulzebos et al 2 found that preoperative intense inspiratory muscle training could prevent pulmonary problems in high-risk CABG patients, implying that maintaining inspiratory muscle strength after surgery is critical for avoiding postoperative morbidity (9).

\section{PATIENT INFORMATION}

The patient was well before 2 months back. When on 20-12-2021, he started pain in the chest which was sudden in onset and referring to shoulder and back associated with palpitations and breathlessness, was taken to a private hospital in Hinganghat by ambulance where he was asked to do ECG, was referred to the civil hospital located in Sewagram. On the same day where he was admitted for 5days and was under medications. As his symptoms were relieved the patient was discharged and was prescribed medications as above mentioned for a period of 1 month. Then he came to AVBRH, Sawangi on 28-01-2022, and according to the patient, there was a certain blockage in the heart as he was asked to do angiography and was advised for surgery. On 29-01-2022, the patient had undergone several blood tests which was within normal ranges and was under observation till 30-012022. On 31-01-2022, the patient had 
surgery and was under observation in ICU till 03-02-2022 was referred to cardiorespiratory physiotherapy on 01-02-2022. Then was shifted to the ward on 04-02-2022 and there too physiotherapy continued.

\section{Past medical history:}

The patient has a history of hypertension mellitus-2

The patient has a history of diabetes

The patient was tested positive for CoVID-19 on 16-01-2022

Differential diagnosis:- pericarditis

$$
\begin{aligned}
& \text { angina pectoris } \\
& \text { myocardial infarction }
\end{aligned}
$$

\section{Surgical history:}

The patient had on-pump CABG with 4 grafts (LIMA to D1, LIMA to LAD, SVG-OM, SVG-PDA) under general anesthesia on 31-01-2022.

Drug history:

- T. pan 40mg od

- T. clopitab A $150 \mathrm{mg}$ od

- T. results $20 \mathrm{mg}$ od

- T. vit C bd

- T. folic acid od

- T. lasilactone $25 \mathrm{mg}$ bd

- T. digoxin $0.25 \mathrm{mg}$ od

- T. alprax $0.5 \mathrm{mg}$ hs

- Syp. Lactulose $15 \mathrm{ml} \mathrm{hs}$

\section{Personal History:}

- Sleep- unaltered

- Appetite- unaltered

- Diet- mixed

- Bowel- constipation

- Bladder- intact

- Addictions- tobacco chewing:-20years

- Alcohol:- 20years

Family history: Not relevant

Environmental history: Patient lives in kachha house with Indian style toilet.

Socio-economic status: According to Modified Kuppuswamy Scale patient lies under lower class.
CLINICAL FINDINGS:

General examination:

Built: Mesomorph

Posture: Forward head posture, Rounded shoulder

Height: $180 \mathrm{~cm}$

Weight: $65 \mathrm{~kg}$

BMI: $20.1 \mathrm{~kg} / \mathrm{m}^{2}$

Temperature: Patient was afebrile

Pulse rate: 82 beats $/ \mathrm{min}$

SPO2: $100 \%$

BP: $110 / 80 \mathrm{mmhg}$

RR: 21 breaths/min

\section{Systemic examination: Inspection}

- Shape of chest- normal

- Breathing pattern- abdominal-thoracic

- I:E ratio- $1: 1$

- Incision/ scar/ wounds- midsternal

\section{Palpation}

- Trail sign- absent

- Chest expansion:- axillary level- 79$80 \mathrm{~cm}$

nipple level- $89-91 \mathrm{~cm}$

xiphisternum- 78-79cm

Chest symmetry- symmetrical

Thoracic configuration- AP diameter is less than transverse diameter

Extension of scar- $18 \mathrm{~cm}$ in length

Tactile fremitus- present

Percussion- resonant over the $b / l$ lung field

Auscultation- air entry B/L equal

Provisional diagnosis:- Myocardial Infarction

\section{INVESTIGATIONS:}

ECG:- showed ST elevation with anterior wall MI

On January 28, 2022, an angiography revealed triple vascular disease, with $80 \%$ of the lesion in the left anterior descending artery, $80 \%$ in the left circumflex artery, 99 percent in the mid right coronary artery, and $90 \%$ in the distal coronary artery. 

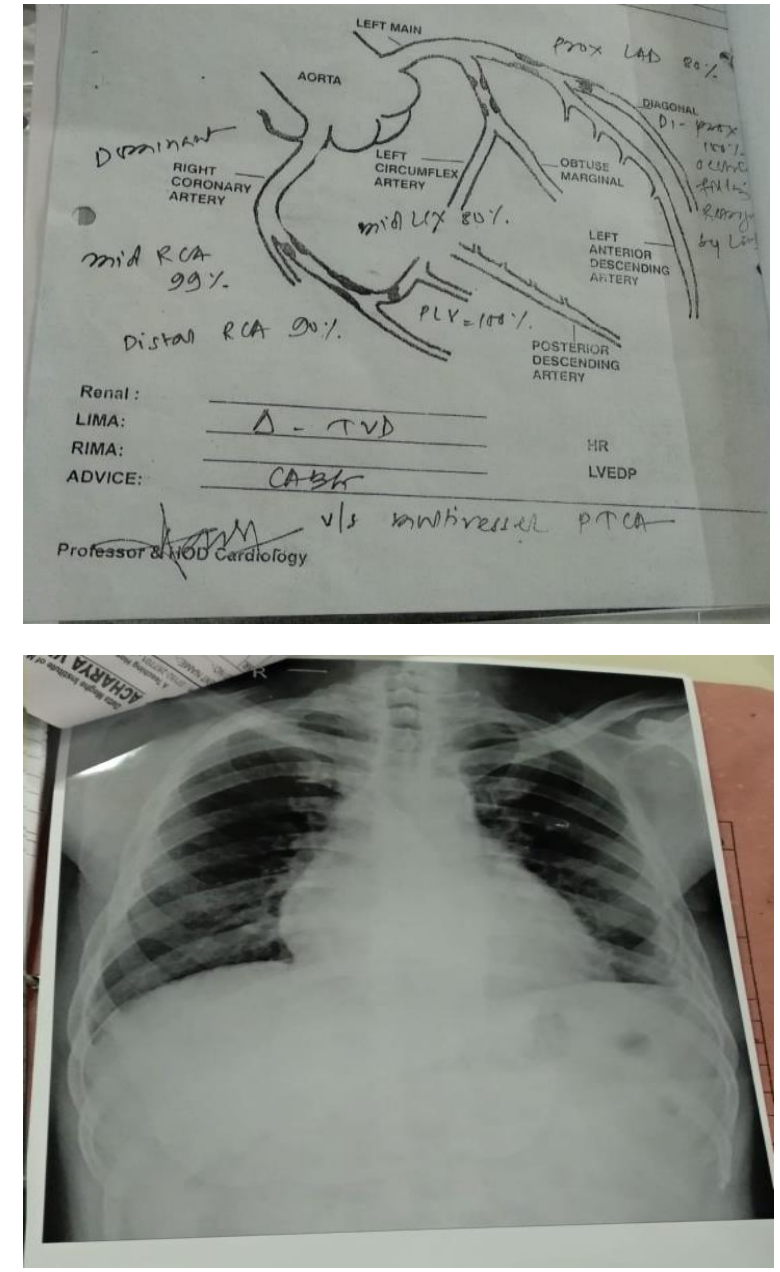

- Chest X-ray PA view

- 2-D Echo showed ejection fraction:$45 \%$

- ABG:-

on 01-02-2022:-

compensated respiratory acidosis

on 02-02-2022:- compensated respiratory acidosis

Blood test:- on 29-01-2022 (kft, lft, cbc, coagulation profile) were within normal ranges.

\section{Outcome Measures:-}

VAS: - on activity $=03 / 10$ on rest $=0 / 10$

NYHA: class ii- limited physical activity, tolerable at rest, but normal physical activity induces weariness, palpitation, or dyspnea.

Diagnosis: Post operative CABG complication of Coronary Artery Disease
International Classification of Functioning, Disability and Health:-

Body function:- Pain (over incision site while doing upper limb mobility)

Structural function:- Cardiovascular system and Respiratory system

Activity limitation and participation restriction:-

Lifting and carrying objects and Walking

Problem list:-

1. reduced lung capacity

2. weakness of pectoralis, abdominal muscles

\section{THERAPEUTIC INTERVENTION:}

\section{Goals:}

Short-term goals:-

- Physical reconditioning sufficient for resumption of customary activities.

- Inform the patient and family about the disease process psychological support throughout the early stages of recovery

\section{Long-term goals:-}

- Identify and treating risk factors that influence the progression of disease (diet, alcohol intake, tobacco chewing)

- Teaching and reinforcing prognosisimproving health behaviours

- Improving physical conditioning and making it easier to return to work and other activities.

\section{Program should include:-}

- Warm up phase- only bed mobilization initially; as fitness improves patient can sit stand by the bed trunk movements can be given and stretching can be given.

- Aerobic phase- walking

- Cool down phase- ROM exercises, stretching

- Monitoring for exercise response:giddiness, chest pain, palpitations

- FITT criteria

- Frequency 2-3times/day

- Intensity RHR+30bpm 
- Timing 5-20 mins intermittent bouts of activity $<5$ mins interspersed with rest periods

- Type- sitting/standing functional activities, ROM exercises, progressive walking, climbing stairs, self care, ADLs.

\section{To improve chest compliance:-}

- Maintenance of lung hygiene by giving relaxed deep breathing exercises and breathing control

- Incentive spirometry

- Splinted huffing/coughing

\section{To prevent DVT:-}

- Ankle toe movements

- AROM exercises of lower limbs

\section{Mobilization:-}

- Dynamic exercises of quadriceps, hamstrings

\section{DISCUSSION}

Maximum lung inflations increase surfactant production, improve compliance and oxygenation, and may help with atelectasis zone re-expansion (2). Incentive spirometry is commonly used in conjunction with physical therapy in the United Kingdom, but it is less commonly used in the United States (2). The decrease in pulmonary function and, as a result, FVC and muscle strength following CABG has been attributed to a number of factors (3). Anaesthesia, analgesics, surgical stress, discomfort, diminished ventricular function, phrenic nerve injury, cardiovascular medicines, extracorporeal circulation (EC), and the position of the drains are all recorded variables(3). The pain from the thoracotomy restricts mobility and respiration. Muscle and ligament retraction, pleural and septal nerve stimulation via thoracic drains, and uncommon rib fractures, as well as skin, muscle, and pleura cutting, can all result in significant postoperative pain (3). Pain may lead to decreased cough efficacy due to rigidity of the thoracic wall, which is the primary mechanism for emptying secretions from the tracheobronchial tree (3). Our intervention, which included respiratory physical therapy, is likely to have resulted in a less severe decline in peak flow, as well as a lower incidence of pleural effusions, atelectasis, and pneumonia (8).

\section{CONCLUSION}

The above studies suggest that early rehabilitation of physiotherapy contributed to the improvement of the patient's functional objectives, which are a significant understanding of a good recovery.

Author's Contribution: For the concept, assessment, and evaluation, data collection analysis, and interpretation of the data, each author made the best contribution.

Informed Consent: Proper consent was taken from the patient's son for writing the case report.

Funding: There is no financial conflicts of interest to disclose.

\section{Acknowledgement: None}

\section{Conflict of Interest: None}

\section{REFERENCES}

1. Hulzebos EH, Van Meeteren NL, De Bie RA, Dagnelie PC, Helders PJ. Prediction of Postoperative Pulmonary Complications on the Basis of Preoperative Risk Factors in Patients Who Had Undergone Coronary Artery Bypass Graft Surgery. Phys Ther. 2003 Jan 1;83(1):8-16.

2. Crowe JM, Bradley CA. The Effectiveness of Incentive Spirometry With Physical Therapy for High-Risk Patients After Coronary Artery Bypass Surgery. Phys Ther. 1997 Mar 1;77(3):260-8.

3. Moreno AM, Castro RR, Sorares PP, Anna MS, Cravo SL, Nóbrega AC. Longitudinal evaluation the pulmonary function of the pre and postoperative periods in the coronary artery bypass graft surgery of patients treated with a physiotherapy protocol. J Cardiothorac Surg. 2011 Apr 27;6(1):62. 
4. Yánez-Brage I, Pita-Fernández S, JufféStein A, Martínez-González U, PértegaDíaz S, Mauleón-García Á. Respiratory physiotherapy and incidence of pulmonary complications in off-pump coronary artery bypass graft surgery: an observational follow-up study. BMC Pulm Med. 2009 Jul 28;9(1):36.

5. Supervised Moderate Intensity Exercise Improves Distance Walked at Hospital Discharge Following Coronary Artery Bypass Graft Surgery- A Randomised Controlled Trial - ScienceDirect [Internet]. [cited 2022 Feb 8]. Available from: https://www.sciencedirect.com/science/artic le/abs/pii/S1443950607008931

6. Postoperative physical therapy after coronary artery bypass surgery. | American Journal of Respiratory and Critical Care Medicine [Internet]. [cited 2022 Feb 8]. Available from: https://www.atsjournals.org/doi/abs/10.1164 /ajrccm.152.3.7663809

7. Haeffener MP, Ferreira GM, Barreto SSM, Arena R, Dall'Ago P. Incentive spirometry with expiratory positive airway pressure reduces pulmonary complications, improves pulmonary function and 6-minute walk distance in patients undergoing coronary artery bypass graft surgery. Am Heart J. 2008 Nov 1;156(5):900.e1-900.e8.

8. Herdy AH, Marcchi PLB, Vila A, Tavares C, Collaço J, Niebauer J, et al. Pre- and Postoperative Cardiopulmonary Rehabilitation in Hospitalized Patients Undergoing Coronary Artery Bypass Surgery: A Randomized Controlled Trial. Am J Phys Med Rehabil. 2008 Sep;87(9): 714-9.

9. Stein R, Maia CP, Silveira AD, Chiappa GR, Myers J, Ribeiro JP. Inspiratory Muscle Strength as a Determinant of Functional Capacity Early After Coronary Artery Bypass Graft Surgery. Arch Phys Med Rehabil. 2009 Oct 1;90(10):1685-91.

How to cite this article: Ubare T, Rathi N, Jaiswal L. Postoperative physical therapy following coronary artery bypass surgery - a case report. Int J Health Sci Res. 2022; 12(3): 138-143. DOI: https://doi.org/10.52403/ijhsr. 20220319 